\title{
Systematic review of the top 10 ranked spine articles in the last 10 years [2011-2021]
}

\author{
Sihyong J. Kim ${ }^{1,2,3}$, Ralph J. Mobbs ${ }^{1,2,3}$ \\ ${ }^{1}$ NeuroSpine Surgery Research Group (NSURG), Sydney, Australia; ${ }^{2}$ NeuroSpine Clinic, Prince of Wales Private Hospital, Randwick, Australia; \\ ${ }^{3}$ Faculty of Medicine, University of New South Wales (UNSW), Sydney, Australia \\ Contributions: (I) Conception and design: Both authors; (II) Administrative support: RJ Mobbs; (III) Provision of study material or patients: RJ Mobbs; \\ (IV) Collection and assembly of data: Both authors; (V) Data analysis and interpretation: Both authors; (VI) Manuscript writing: Both authors; (VII) \\ Final approval of manuscript: Both authors. \\ Correspondence to: Sihyong J. Kim. NeuroSpine Clinic, Suite 7, Level 7 Prince of Wales Private Hospital, 320-346 Barker St, Randwick, New South \\ Wales, 2031, Australia. Email: usujake@gmail.com.
}

Background: Spine-related research continues to evolve rapidly and in the paradigm of increasing data, evidence-based practice becomes imperative. Citation-based rankings are thus critical in allowing clinicians to quickly ascertain the importance and value of a study. The purpose of this article is to report on the 10 most cited articles in the field of spine surgery over the last 10 years to provide an insight into the direction of research and clinical endeavors.

Methods: Google Scholar was searched (1st April 2021) using an algorithm that sorts all cited spine surgery publications based on the number of citations per year. The top 10 most cited articles were identified. Information including journal, publication title, published year, subspecialty, and purpose of the study were compiled.

Results: The top 10 publications ranged from 471 to 66 citations, with yearly citations ranging from 67 to 14 . Eight articles directly related to lumbar fusion, 2 related to 3D Printing in spinal surgery, and one article on robotic surgery. There were 4 retrospective studies, 1 randomized controlled trial (RCT), and 2 systematic reviews. 3 of the papers related to decision making in surgery, 4 on outcomes of surgery, and 3 on innovations in surgery. The journal that appeared most frequently in the top 10 list was the Fournal of Spine Surgery.

Discussion: Novel surgical approaches or management strategies are almost always a manifestation of advancements in clinical and basic science research. Algorithm-based identification of highly cited articles provides an effective and prompt avenue for evidence-based medicine. Our ranking found a predominance of publications related to lumbar spinal fusion. Several articles in the top 10 provide an in-depth discussion on novel surgical techniques and technologies that define the current epoch of innovations in spine surgery.

Keywords: Spine surgery; lumbar fusion; citation analysis; citation ranking

Submitted May 10, 2021. Accepted for publication May 28, 2021.

doi: 10.21037/atm-21-2393

View this article at: https://dx.doi.org/10.21037/atm-21-2393

\section{Introduction}

The surgeon's approach and understanding of spinal disorders have rapidly expanded due to significant contributions made in both clinical and basic science research. Seminal studies are highly cited and represents a change or refinement of perspectives towards certain surgical techniques and management strategy. In essence, these studies serve as the fulcrum upon which clinical expertise can be balanced with empirical evidence, often derived from randomized controlled trials (RCTs), systematic reviews (SRs), and meta-analyses (MAs). 
Identification

Algorithmic ranking

Screening

Included

Figure 1 Search strategy and screening process for the systematic review of the top 10 most cited spine articles, 2011-2021.

However, the rapid growth of the scientific literature can make it difficult for clinicians to distinguish between spurious and valid clinical data. Furthermore, critical appraisal of the various publications provided by databases such as PubMed, which traditionally does not sort or rank studies based on 'impact' or 'seminality', makes the process of evidence-based medicine (EBM) time-consuming for the clinician. In this paradigm, the introduction of machinelearning-based algorithms such as in the establishment of a citation-based rank is crucial to identify those works that have had the greatest intellectual and clinical influence.

"Citations" or "citation count" is generated when a peerreviewed publication references another body of work. Powerful derivatives of machine-learning algorithms are capable of (I) generating citation count and (II) sorting searches based on these numbers. To the best of our knowledge, citation counts represent the only objective way of determining the influence and impact of an article in a convenient and relatively quick manner. There are many avenues by which citation analyses can be conducted. For example, the Institute for Scientific Information (ISI), utilizes the "Science Citation Index Expanded" system to track citation information for these manuscripts. However, Google Scholar presents a far greater and more comprehensive capture of published research using the powerful algorithms of Google to identify research from all sources. Scopus and Web of Science are also good alternatives; however, these databases are not entirely inclusive of the overall literature. The purpose of this study was to identify and characterize the most frequently cited articles in the field of spine surgery. We present the following article in accordance with the Preferred Reporting Items for Systematic Reviews and MetaAnalyses (PRISMA) reporting checklist (available at https://dx.doi.org/10.21037/atm-21-2393).

\section{Methods}

Figure 1 outlines an overview of the search process for this review. A broad search using terms, (Spinal Surgery) OR (Spinal Fusion) OR (Lumbar Fusion) OR (Cervical Spine) OR (Spinal Fixation), was executed on the Google Scholar database, leading to 247,000 total results. We utilized an algorithm that indexed all bibliometric data including the total number of citations and citation count per year generated by Google Scholar's in-built citation searching function. The resulting data frame containing all 247,000 results and their associated citation count was algorithmically organized and sorted in order of decreasing citations per year. Additional information regarding the author, year of publication, the title of publication, and published journal was collated and appended to the existing data frame. Additional lines of command were executed to filter the papers for recency, screening for papers that were published in the last 10 years. The top 200 papers were briefly examined for an overview of the literature and to ensure quality control of our search terms. Finally, the top 10 papers with the highest citations per year were selected for review (1-10).

The top 10 studies were classified into various categories to provide a high-level overview of the most impactful and influential papers in the recent spine literature. Firstly, each study was grouped according to type of study design e.g., RCTs, retrospective cross-sectional studies, SRs, MAs, etc. Secondly, the studies were classified according to the specific spinal surgery or clinical condition that was of focus e.g., lumbar interbody fusion (LIFs), pedicle/ posterior fixation $(\mathrm{PF})$, etc. Lastly, in an attempt to provide an analysis of the purpose of each study, each study 
Table 1 Top 10 spine surgery publications ranked according to number of citations per year (cit/year)

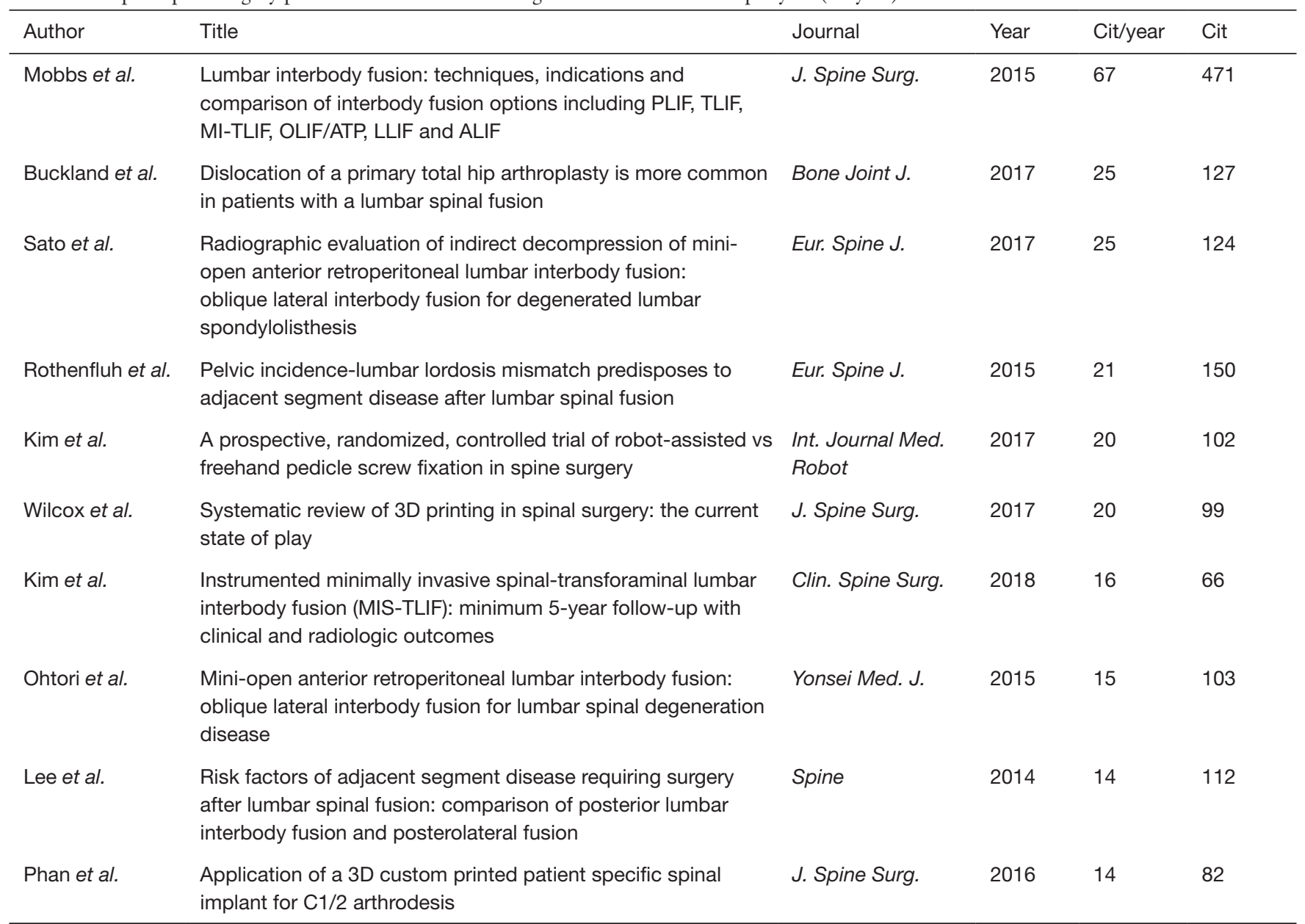

Cit, citations; Cit/year, citations per year.

was indexed according to three overarching themes: (I) surgical decision making, (II) innovations in surgery, and (III) outcomes of surgery. Articles were classified under 'surgical decision making' if it encompassed data relating to patient risk factors that warrant spinal surgery, details the technicalities of the surgeon's thought-process during and after surgery, or discusses the management process of spinal surgical cases. Articles classified under 'innovations in surgery' discuss technological advancements that aids surgeon's decision-making or surgical workflow e.g. the use of $3 \mathrm{D}$ printing, neuronavigational software, robot-assisted surgeries, synthetic grafts, and novel materials for cages and screws. These groups were not designed to be mutually exclusive, that is, a study could affiliate with many groups.

\section{Results}

Table 1 lists the top 10, highest cited papers on the topic of spine surgery between the years 2011-2021. The top-ranked paper by Mobbs et al. had the highest total number of citations of 471 with an average of 67 citations per year (1). The number of citations per year across the top 10 ranged from 67 to 14 . The range of total citations was 471 to 66 . The difference in total citations between the rank 1 and 2 papers was 344 . The mean number of citations in the top 10 was 143.6 with a standard deviation of 111.4. Amongst the top 10, only 1 paper was included in the top $25 \%$ quartile of citations per year, 5 papers in the next highest quartile, and the remaining 4 papers in the bottom two quartiles. Three papers were from the Journal of Spine 
Table 2 Frequency of each study in each category

\begin{tabular}{ll}
\hline Category & No. of studies \\
\hline Randomized controlled trials & 1 \\
Systematic reviews & 2 \\
Meta-analysis & 0 \\
Retrospective cross-sectional/case-control/ & 4 \\
cohort studies & \\
Prospective study & 1 \\
Radiographical evaluation & 1 \\
Case report & 1 \\
Lumbar interbody fusion & 8 \\
Cervical fusion & 1 \\
Pedicle screw fixation & 2 \\
Decision making in surgery & 3 \\
Surgical innovations \& new surgical technology & 3 \\
Outcomes of surgery & 4 \\
Adjacent segment disease & 2 \\
Spondylolisthesis & 3 \\
Spinal stenosis & 2 \\
Facet arthropathy & 1 \\
Low back pain & 4 \\
Non-spondylotic (trauma, cancer) & \\
\hline
\end{tabular}

Surgery, with a mean total citation of 217, 2 papers from the European Spine fournal, with a mean citation of 127 and the remaining papers were each from the Bone and Foint Fournal, the International Fournal of Medical Robotics, Clinical Spine Surgery, the Yonsei Medical Fournal, and Spine.

Table 2 lists the number of studies in each category according to the type of study, specific surgery in focus, overarching themes, and relevant pathologies. See also Figure 2 for a visual representation of this data. There were at least 2 articles in our study that were of Level 1 evidence criteria. 4 of the top 10 rankings were retrospective clinical studies. Thereafter, 2 of the top 10 studies were SRs. There was 1 RCT, 1 prospective follow-up of patients following lumbar fusion, 1 post-operative radiographical evaluation, and 1 case report of a novel 3D-printed spinal prosthesis for cervical fusion. There were no MAs included in the top 10 .

The majority (8) of the papers in the top 10 evaluated some form of LIF. The rank 1 paper was a systematic review that discussed the technicalities, indications, disadvantages, and recommendations for all known forms of LIF, i.e. posterior LIF (PLIF), transforaminal LIF (TLIF), lateral LIF (LLIF/XLIF), oblique LIF (OLIF), and anterior LIF (ALIF)(1). The rank 2 paper investigated the effect of an unspecified LIF approach in predisposing patients to total hip arthroplasty (THA) dislocation (2). This paper also evaluated the effect of multiple segmental fusion on increasing the likelihood of THA dislocation(2). The rank 3 study was a radiographic evaluation of deformity correction in 20 patients who underwent OLIF (3). The rank 4 study evaluated the relationship between sagittal balance parameters such as pelvic incidence to adjacent segment disease following LIFs of unspecified approach between the L2-S1 level (4). Both rank 5 and 8 papers focused on PLIFs with $360^{\circ}$ fusion using additional pedicle screw instrumentation $(5,8)$. The rank 5 paper was also the only RCT that comparatively explored fusion outcomes following robot-assisted and free-hand pedicle screw fixation (5). The only none-LIF paper that was identified, was a case report detailing the first known use of a novel 3D printed, patient-specific prosthesis for C1-2 arthrodesis (10).

There was a relatively even distribution of the three overarching themes we have identified for this review. 3 out of 10 papers related to the factors that contributed to the decision-making process in spinal surgery, elucidating various risk factors for intra- and postoperative complications or discussing the intricacies and nuances of the surgeon's thought process when planning and executing spinal surgeries $(1,4,9)$. Three papers discussed various forms of surgical innovations with 2 papers discussing $3 \mathrm{D}$ printed, patient-specific prosthesis or drill guides and 1 paper exploring the use of robot-assisted surgeries in pedicle screw instrumentation $(5,6,10)$. Finally, 4 papers $(2,3,7,8)$ evaluated postoperative outcomes of surgery using a variety of techniques ranging from radiological parameters and patient-reported outcome measures (PROMs) such as the Oswestry Disability Index (11) and the Visual Analogue Scale (12).

A variety of spinal pathologies were covered in the top 10 most heavily cited papers. The majority of studies (4) discussed the role of LIFs in treating the degenerative spine, especially those with symptomatic spondylolisthesis. 3 of the 10 studies revolved around the post-operative complication of adjacent segment disease (ASD) $(4,7,9) .2$ papers had lumbar spinal stenosis (LSS) as a major criterion for inclusion in the patient sample $(5,8)$. No highly cited and influential paper discussed the surgical treatment of 


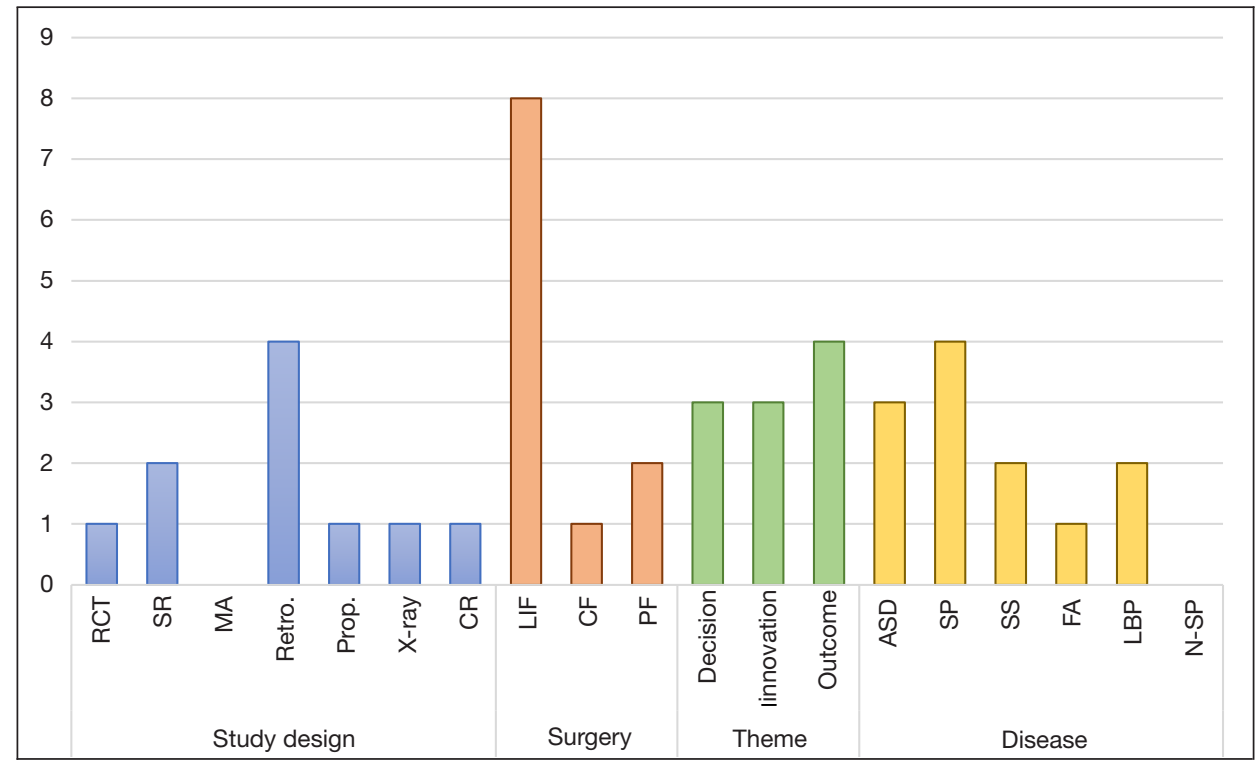

Figure 2 Top 10 articles according to study design, type of spinal surgery, overarching themes (decision making in surgery, innovations in surgery, outcomes in surgery) and pathology. The y-axis indicates the number of publications that were found in each category. RCT, randomized controlled trials; SR, systematic review; MA, meta-analyses; CR, case report; LIF, lumbar interbody fusion; CF, cervical fusion; $\mathrm{PF}$, posterior fixation with pedicle screws; ASD, adjacent segmental disease; SP, spondylolisthesis; SS, spinal stenosis; FA, facet arthropathy; LBP, low back pain; N-SP, non-spondylotic diseases.

non-spondylotic spinal pathologies such as trauma or cancer.

\section{Discussion}

The surgical management of spinal disorders requires a multi-disciplinary and evidence-based approach. New surgical approaches or management strategies that improve patient outcomes are almost always a corollary of scientific and clinical investigations that expand on the academic knowledge of a given field. Allowing surgeons and scientists to easily identify these significant contributions is important in providing a direction on where the specialty is heading. Furthermore, considering the rise of numerous available data in the surgical management of patients, clinicians need to be able to distinguish between spurious and valid findings. However, critical appraisal of the broad scientific literature is often time-consuming and not user-friendly, especially given that validated databases such as PubMed do not allow users to quickly sort searches based on relevant bibliometric data such as the number of citation counts and journal impact factors (13). In this paradigm, machine-learning-driven processes that identify influential and reliable papers are critical in improving patient outcomes (14). As such, this study aimed to evaluate the most impactful and relevant literature on spine surgery in the last 7 years using an algorithm that sorts studies based on citation count.

Our ranking recognizes some emerging seminal papers of spine surgery care; the primary example is the article by Mobbs et al. (1) that established the thought processes involved in determining the approach for interbody fusion based on the evolution of multiple approaches and anatomical corridors. This comprehensive systematic review provides not only information regarding the technicalities of each LIF approach but also sheds light on the importance of considering various factors such as the segmental level, possible complications/risks, and surgeon competency in guiding the surgeon's decision making, from beginning to end. Most articles in the top 10 are related to lumbar fusion; therefore, this intervention is at the forefront of clinicians' and researchers' minds. Furthermore, 3D printing has evolved rapidly and has been well integrated into the surgical workflow over the last 5 years and this is well recognized by 2 papers $(6,10)$ in the top 10 , both articles by the NSURG research team in Sydney, Australia.

Three of the most highly cited papers on our list illustrated the versatility and potential of novel surgical 
innovations. It is interesting to consider that the reason these studies are highly cited may perhaps be due to the excitement that surrounds the dynamic interplay between advancements in the industry and the pioneers of spinal surgery. Indeed, the arena for discussing these innovations has been rapidly expanding with a 50-year review finding that out of 1,162 publications found with the search terms 'innovation', 'new technology', and 'spine surgery', $83 \%$ were published after 2006 (15). These technologies are unique, ranging from $3 \mathrm{D}$-printed prosthesis and drill guides, surgical navigation, robot-assisted surgeries, and sensor-based 'wearables'. Furthermore, the 2 papers on $3 \mathrm{D}$ printing found in our list could represent the increasing popularity of patient-centered or personalized medicine (16). As discussed by the authors in these papers, $3 \mathrm{D}$ printing provides an avenue through which intraoperative duration and rates of complications may be decreased as a result of this patient-centered-ness (6). It is also a versatile tool that can easily be incorporated into the surgical workflow.

Undeniably, the quality of spine research continues to improve with health care reform demanding the emphasis on evidence-based medicine and comparative effectiveness research in spine care, and these types of studies will be increasingly important in advancing treatment. Indeed, 4 papers in the top 10 echo this philosophy, utilizing empirical data from clinical trials to evaluate the outcomes of lumbar fusion surgeries. In particular, the $2^{\text {nd }}$ most highly cited paper on our list provides evidence that patients who receive THAs after receiving lumbar fusion have an increased risk of hip dislocation (2). Furthermore, the authors find that this correlation is magnified with increasing levels of fusion (2). This type of empirical evidence is valuable in the management of the aging population, where it is becoming increasingly likely that patients will present with multiple co-morbidities and thus require many different surgeries. Surgeons must be able to understand both the immediate and long-term consequences of surgeries by referring to highly influential and relevant studies such as this. Furthermore, a comparative analysis of different surgical approaches such as the one by Kim et al. is critical in reforming and ultimately improving the current state of spinal surgery. Here, the authors employed an RCT to determine that robot-assisted pedicle screw insertion resulted in less facet joint violation and superior insertion angles over the traditional, free-hand approach (5). Furthermore, the robot-assisted group demonstrated a proximal facet joint accuracy of $100 \%$ compared to $84 \%$ in the free-hand group (5). This influential paper is critical in cementing the direct correlation between advancements in technology and improved patient outcomes.

According to our rank, the Journal of Spine Surgery generated the most number of highly cited articles in the last decade. It is important to note that evaluating the quality and impact of a journal based on citation count is not entirely valid. As suggested by Murray et al. there are inherent differences between the frequency of publications between journals and as a result, bimonthly journals that publish a greater number of articles than less frequent journals have a greater chance of being cited (17). Furthermore, journals and articles that have been in circulation for a longer time will generally have accumulated more citations. For these reasons, we decided to (I) rank studies based on the number of citations per year and (II) utilize Google Scholar rather than Scopus or Web of Science which is dominated by journals with high impact factors such as the Spine Journal.

There were several limitations of this review. One was that using Google Scholar alone is not enough to cover the entire literature on spine surgery. To the best of our knowledge, there are only two other databases, Scopus and Web of Science that allow users to 'sort' by citation count. A study has found that using just one of these three databases e.g., Scopus would find $76 \%$ of all citing references in a given field (13). With the addition of another database e.g., Google Scholar, the user would find a total of $94 \%$ of all citing references (13). However, a manual search in all 3 databases revealed several flaws that made it difficult to conduct such a comprehensive search. Firstly, due to the inherent differences in the algorithms employed by each database, citation counts were inconsistent across the three databases. For example, Scopus and Web of Science only count a citation if a study has been referenced by an original journal article whilst Google Scholar is a broader, more encompassing database that incorporates data from books and other academic sources (13). Secondly, Scopus and Web of Science do not contain publications from many distinguished journals of lower impact, which naturally disadvantages credible and impactful papers.

Furthermore, although very broad search terms encompassing a variety of topics within spinal surgery were used, it is impossible to consider that every relevant article was discovered. This is perhaps related to the inherent shortcomings of the Google Scholar search algorithm which functions differently from other known databases such as PubMed and Medline. Additionally, studies focusing on 
basic science topics that apply vaguely to spine surgery were excluded. For example, consider the study by Wright et al. that lists the bone mineral density values in the lumbar spines of the US population (18). This paper has been cited over 1,000 times and would mask many important studies in the relatively smaller sub-specialty of spinal surgery. Whilst basic science studies such as the one by Wright et al. is critical in allowing surgeons understand relevant patient demographics, it pertains to a broader field of 'bone health' and 'aging.' As such, these papers were excluded to focus on studies that had specific relevance and applicability to 'spine surgery'.

There is contention in the field of biomedical information regarding whether citation count is an accurate reflection of the quality and reliability of a study $(19,20)$. Furthermore, citation counts are subjected to the "obliteration by incorporation" bias. The theory behind this bias states that relatively old publications become heavily incorporated into the language and jargon of the field's current body of knowledge and as a result, are cited with reduced frequency and vigour (21). This is one of the contributing reasons as to why our algorithm sorted the studies based on the number of citations per year. If the total number of citations was used, it is possible that the "obliteration by incorporation effect" would have manufactured an artificial increase in citations for more recent articles, giving them an advantage over older, 'seminal' papers (17).

Despite all the above limitations, citation counts are the only objective measure of a paper's influence in advancing the body of knowledge in a given field. By categorically reviewing the top 10 most cited articles in the field of spine surgery, we are able to shed light on potential landmark publications and the respective authors who are responsible for directing evidence-based care of spine surgery patients in the last decade. This review also serves to highlight the technologies, innovations, evaluation models, and surgical approaches that are the drivers of advancements in this unique and dynamic sub-specialty.

\section{Conclusions}

Using an algorithm-based search, the top 10 most highly cited papers in the field of spinal surgery in the last 10 years were analyzed for review. These influential and highly relevant studies discussed factors that aid surgical decision-making, current innovations in spinal surgery, and the evaluation of postoperative outcomes. These studies reflect a critical facet of the modern surgeon's thought process in the current epoch of spine surgery.

\section{Acknowledgments}

The NeuroSpine Surgery Research Group (NSURG) aided with manuscript production. The NeuroSpine Clinic, Sydney Australia, provided clinic assistance for authors. Funding: None.

\section{Footnote}

Reporting Checklist: The authors have completed the PRISMA reporting checklist. Available at https://dx.doi. org/10.21037/atm-21-2393

Conflicts of Interest: Both authors have completed the ICMJE uniform disclosure form (available at https://dx.doi. org/10.21037/atm-21-2393). The authors have no conflicts of interest to declare.

Ethical Statement: Both authors are accountable for all aspects of the work in ensuring that questions related to the accuracy or integrity of any part of the work are appropriately investigated and resolved.

Open Access Statement: This is an Open Access article distributed in accordance with the Creative Commons Attribution-NonCommercial-NoDerivs 4.0 International License (CC BY-NC-ND 4.0), which permits the noncommercial replication and distribution of the article with the strict proviso that no changes or edits are made and the original work is properly cited (including links to both the formal publication through the relevant DOI and the license). See: https://creativecommons.org/licenses/by-nc-nd/4.0/.

\section{References}

1. Mobbs RJ, Phan K, Malham G, et al. Lumbar interbody fusion: techniques, indications and comparison of interbody fusion options including PLIF, TLIF, MI-TLIF, OLIF/ATP, LLIF and ALIF. J Spine Surg 2015;1:2-18.

2. Buckland AJ, Puvanesarajah V, Vigdorchik J, et al. Dislocation of a primary total hip arthroplasty is more common in patients with a lumbar spinal fusion. Bone Joint J 2017;99-b:585-91.

3. Sato J, Ohtori S, Orita S, et al. Radiographic evaluation of indirect decompression of mini-open anterior 
retroperitoneal lumbar interbody fusion: oblique lateral interbody fusion for degenerated lumbar spondylolisthesis. Eur Spine J 2017;26:671-8.

4. Rothenfluh DA, Mueller DA, Rothenfluh E, et al. Pelvic incidence-lumbar lordosis mismatch predisposes to adjacent segment disease after lumbar spinal fusion. Eur Spine J 2015;24:1251-8.

5. Kim HJ, Jung WI, Chang BS, et al. A prospective, randomized, controlled trial of robot-assisted vs freehand pedicle screw fixation in spine surgery. Int J Med Robot 2017;13.

6. Wilcox B, Mobbs RJ, Wu AM, et al. Systematic review of 3D printing in spinal surgery: the current state of play. $\mathrm{J}$ Spine Surg 2017;3:433-43.

7. Kim JS, Jung B, Lee SH. Instrumented Minimally Invasive Spinal-Transforaminal Lumbar Interbody Fusion (MISTLIF): Minimum 5-Year Follow-Up With Clinical and Radiologic Outcomes. Clin Spine Surg 2018;31:E302-9.

8. Ohtori S, Orita S, Yamauchi K, et al. Mini-Open Anterior Retroperitoneal Lumbar Interbody Fusion: Oblique Lateral Interbody Fusion for Lumbar Spinal Degeneration Disease. Yonsei Med J 2015;56:1051-9.

9. Lee JC, Kim Y, Soh JW, et al. Risk factors of adjacent segment disease requiring surgery after lumbar spinal fusion: comparison of posterior lumbar interbody fusion and posterolateral fusion. Spine (Phila $\mathrm{Pa} 1976$ ) 2014;39:E339-45.

10. Phan K, Sgro A, Maharaj MM, et al. Application of a 3D custom printed patient specific spinal implant for C1/2 arthrodesis. J Spine Surg 2016;2:314-8.

11. Fairbank JC, Pynsent PB. The Oswestry Disability Index.
Spine (Phila Pa 1976) 2000;25:2940-52; discussion 2952.

12. Mannion AF, Balagué F, Pellisé F, et al. Pain measurement in patients with low back pain. Nat Clin Pract Rheumatol 2007;3:610-8.

13. Bakkalbasi N, Bauer K, Glover J, et al. Three options for citation tracking: Google Scholar, Scopus and Web of Science. Biomedical Digital Libraries 2006;3:7.

14. Bian J, Morid MA, Jonnalagadda S, et al. Automatic identification of high impact articles in PubMed to support clinical decision making. J Biomed Inform 2017;73:95-103.

15. Cornwall GB, Davis A, Walsh WR, et al. Innovation and New Technologies in Spine Surgery, Circa 2020: A FiftyYear Review. Front Surg 2020;7:575318.

16. Vicente AM, Ballensiefen W, Jönsson J-I. How personalised medicine will transform healthcare by 2030 : the ICPerMed vision. J Transl Med 2020;18:180.

17. Murray MR, Wang T, Schroeder GD, et al. The 100 most cited spine articles. Eur Spine J 2012;21:2059-69.

18. Wright NC, Looker AC, Saag KG, et al. The recent prevalence of osteoporosis and low bone mass in the United States based on bone mineral density at the femoral neck or lumbar spine. J Bone Miner Res 2014;29:2520-6.

19. Smith R. Beware the tyranny of impact factors. The Journal of Bone and Joint Surgery British volume 2008;90:125-6.

20. Cheek J, Garnham B, Quan J. What's in a Number? Issues in Providing Evidence of Impact and Quality of Research(ers). Qualitative Health Research 2006;16:423-35.

21. Garfield E. 100 citation classics from the Journal of the American Medical Association. Jama 1987;257:52-9.
Cite this article as: Kim SJ, Mobbs RJ. Systematic review of the top 10 ranked spine articles in the last 10 years [2011-2021]. Ann Transl Med 2021;9(13):1090. doi: 10.21037/atm-21-2393 